\title{
The role of long noncoding RNAs in livestock adipose tissue deposition - A review
}

\author{
Lixue Wang ${ }^{1}$, Yuhuai Xie ${ }^{2}$, Wei Chen ${ }^{1}$, Yu Zhang ${ }^{1}$, and Yongqing Zeng ${ }^{1 \text {,* }}$
}

* Corresponding Author: Yongqing Zeng Tel: +86-538-8242478, Fax: +86-538-8242478,

E-mail: yqzeng@sdau.edu.cn

'Shandong Provincial Key Laboratory of Animal Biotechnology and Disease Control and Prevention, College of Animal Science and Technology, Shandong Agricultural

University, Tai'an, Shandong 271018, China

2 Department of Immunology, School of

Basic Medical Sciences, Fudan University,

Shanghai, 200032, China

ORCID

Lixue Wang

https://orcid.org/0000-0001-9704-1527

Yuhuai Xie

https://orcid.org/0000-0001-7280-7098

Wei Chen

https://orcid.org/0000-0002-2901-5156

Yu Zhang

https://orcid.org/0000-0003-1539-9977

Yongqing Zeng

https://orcid.org/0000-0002-0740-2470

Submitted Jan 7, 2021; Revised Mar 5, 2021; Accepted Mar 29, 2021

\begin{abstract}
With the development of sequencing technology, numerous, long noncoding RNAs (lncRNAs) have been discovered and annotated. Increasing evidence has shown that lncRNAs play an essential role in regulating many biological and pathological processes, especially in cancer. However, there have been few studies on the roles of lncRNAs in livestock production. In animal products, meat quality and lean percentage are vital economic traits closely related to adipose tissue deposition. However, adipose tissue accumulation is also a pivotal contributor to obesity, diabetes, atherosclerosis, and many other diseases, as demonstrated by human studies. In livestock production, the mechanism by which $\operatorname{lncRNAs}$ regulate adipose tissue deposition is still unclear. In addition, the phenomenon that different animal species have different adipose tissue accumulation abilities is not well understood. In this review, we summarize the characteristics of $\operatorname{lncRNAs}$ and their four functional archetypes and review the current knowledge about lncRNA functions in adipose tissue deposition in livestock species. This review could provide theoretical significance to explore the functional mechanisms of lncRNAs in adipose tissue accumulation in animals.
\end{abstract}

Keywords: LncRNAs; Adipose Tissue Deposition; Livestock

\section{INTRODUCTION}

Adipose tissue deposition is a crucial economic index for livestock production evaluation and is closely associated with meat quality by influencing the lean meat ratio and intramuscular fat content $[1,2]$. However, in human medical studies, excess fat is a health concern, as abnormal adipose tissue accumulation might lead to obesity, diabetes, atherosclerosis, and other diseases $[3,4]$. In the early embryonic development period and the birth period, the number of adipocytes rapidly increases because of the impact of maternal genetic and nutritional factors. The volume of adipocytes increases relatively quickly in the late embryonic development and growth stage $[5,6]$. Subcutaneous, abdominal, bone, intermuscular and intramuscular tissues are the primary locations of animal adipose depots [7-9]. A high content of lipids in these sites can affect the lean meat ratio and meat quality of animals [10]. Meanwhile, it also leads to obesity and type 2 diabetes in humans [11-13].

Over the past few decades, with the rapid development and great benefits of next-generation sequencing (NGS) technologies, large-scale genome sequencing data can be rapidly generated, which has revolutionized our understanding of genetic informatics. In this field, long noncoding RNAs (lncRNAs) have emerged as important regulators of gene expression in diverse biological contexts. Increasing evidence has demonstrated that lncRNAs play important roles in animal breeding, reproduction and livestock production, especially in the breeding of high-quality meat-producing animals [14,15]. Evidence showed that lncRNAs could regulate muscle growth [16], adipogenic differentiation [17], and adipo- 
genesis of porcine ontramuscular preadipocyte [18].

However, compared with their application in human biomedical studies, the application of lncRNAs in livestock production is much less common. Although previous reviews about lncRNAs in livestock have provided information about the identification, features, and genomic annotation of lncRNAs [19,20], some newer aspects of lncRNA function in improving livestock production have not yet been studied extensively. Thus, in this paper, we review the current studies about the roles of lncRNAs in adipose tissue deposition in livestock species, as well as lncRNA biology, which is expected to benefit animal and food biologists.

\section{CHARACTERISTICS OF LncRNAs}

LncRNAs are defined as noncoding RNAs longer than 200 nucleotides, a cutoff that is used mainly to distinguish lncRNAs from small RNAs such as microRNAs (miRNAs) and transfer RNAs [21]. LncRNAs can be further classified into six different types, namely, long-intergenic ncRNAs (lincRNAs), intronic lncRNAs, antisense lncRNAs, sense lncRNAs, enhancer-associated lncRNAs, and circular lncRNAs [22]. Similar to messenger RNA (mRNAs), lncRNAs are transcribed by RNA polymerase II (Pol II) from genomic loci with chromatin states similar to those of mRNAs. In most instances, lncRNAs are biochemically indistinguishable from mRNAs except for their lack of a translated open reading frame [23]. However, differences have been observed in some lncRNAs that contain introns, can be nonpolyadenylated or are bidirectionally transcribed [24]. Homology and conservation are also important indicators in studying the biological function and relationship with protein-coding genes of lncRNAs.

Typically, evolutionary conservation refers to sequence conservation, which is determined based on similarities between nucleotide or amino acid sequences. A comparison of mammalian transcripts and lncRNAs found in zebrafish revealed that the few instances of significantly conserved sequences were mostly limited to short sequence stretches. Hence, lncRNAs evolve rapidly and often lack orthologs in other species [25]. Secondary and tertiary structures are vital for RNA function [26]. Studies have demonstrated the existence of structured regions within IncRNAs, and multiple sequence alignments have supported the presence of conserved structures $[27,28]$. In addition, while the process of lncRNA production from specific genomic sites may be conserved, the sequence, structure, and function of the products may not be conserved [29]. Finally, the conservation of lncRNA functions in different species is also a relevant point in the field, and is often associated with sequence and structure [30]. Most importantly, the low sequence conservation of lncRNAs across different species has led some to dismiss lncRNAs as transcriptional noise [31]. Despite many predictions from RNA sequencing (RNA-seq) data, few lncRNA orthologs that function across species have been experimentally verified. In addition, data from the UCSC Genome Browser (http://genome.ucsc.edu/) and NONCODE database (http://www.noncode.org/) can help us identify conserved lncRNAs across different species.

Based on transcriptome-wide methods, IncRNAs generally exhibit more specific expression profiles than mRNAs [32]. That is, IncRNA expression is more specific to different cells, tissues and stages of development, and this specific expression can be more tightly regulated than the expression of protein-coding genes. Furthermore, lncRNA expression is correlated with mRNA expression both in cis and in trans, suggesting that lncRNAs may be coregulated in expression networks [23,33]. Many research findings have indicated an essential role of lncRNAs in regulating the development and maintenance of endocrine organs and hormonal signaling, and misregulation of these processes can lead to disease [34]. Because of poor conservation and specific expression, the mechanisms of lncRNAs in regulating biological processes are complex and unique. LncRNAs can direct elements in cis or in trans elements to bind to genes or ribosomal protein complexes, thereby regulating the expression of adjacent or distant genes and acting as molecular guides [35]. In addition, lncRNAs can act as a central platform in different related types of macromolecular components and can play a regulatory role in a variety of biological signal transduction processes.

The function of lncRNAs is associated with their unique subcellular localization patterns. The localization of lncRNAs, mainly nuclear and cytoplasmic localization, has recently become a hot topic in the field. Evidence has shown that compared with mRNAs, IncRNAs are more enriched in the nucleus relative to the cytoplasm [31]. RNA fluorescence in situ hybridization (RNA FISH) has been used to evaluate lncRNA showed the IncRNAs localization, which showed that lncRNAs exhibit many different subcellular localization patterns ranging from defined subnuclear points and nuclear retention to diffuse whole-cell spread. They do not categorically occupy one particular location; rather, lncRNAs are ubiquitous [36].

\section{MECHANISMS OF ADIPOSE TISSUE DEPOSITION}

Adipocytes are derived from multipotent stem cells that are fibroblast-like mesenchymal precursors and can differentiate into adipocytes, myoblasts, osteoblasts, and chondrocytes after receiving signals from specific transcription factors [37, 38]. After signaling by bone morphogenetic protein 4 (BMP4), multipotent fibroblast-like stem cells commit to the adipocyte lineage, and finally differentiate into mature adipocytes 
[39]. The proliferation and hypertrophy of adipocytes are the main factors causing adipose tissue deposition. In pigs and ruminants, hypertrophic adipose tissue predominates after birth. The postnatal growth of adipose tissue results from an increase in the number (hyperplasia) and especially the size (hypertrophy) of adipocytes [40]. Previously, a detailed and systematic endeavor was undertaken to define the transcriptional events regulating preadipocyte differentiation (adipogenesis) and adipocyte function. Peroxisome proliferator-activated receptor $\gamma(\operatorname{PPAR} \gamma)$ and CCAAT enhancer binding proteins $(\mathrm{C} / \mathrm{EBP} \alpha)$ are the master regulators of adipogenesis, which has been confirmed by the vast majority of in vivo and in vitro studies [41,42]. Moreover, other transcription factors ultimately affect adipose tissue deposition through PPAR $\gamma$ and C/EBP $\alpha[43,44]$.

Many signaling pathways modulate the process of preadipocyte differentiation into mature adipocytes. BMPs are a class of conserved signaling molecules in the transforming growth factor- $\beta$ family of proteins. BMP signaling through BMP receptors results in the intracellular phosphorylation and activation of SMAD proteins, which causes the transcription of PPAR $\gamma$ and promotes adipogenesis [45]. The Wnt signaling pathway was once thought to be a negative regulator of adipose differentiation. However, wnt5b could partially inhibit the canonical Wnt/ $\beta$-catenin signaling pathway and promote adipocyte differentiation [46]. In addition, the hedgehog [47], and notch [48] signaling pathways could also regulate adipose tissue deposition.

\section{THE FUNCTION OF LncRNAs IN ADIPOSE TISSUE DEPOSITION}

LncRNAs are involved in the mechanisms of adipose tissue deposition by influencing the adipogenesis and lipid metabolism. On the one hand, the proliferation and hypertrophy of adipocytes are the main processes of adipogenesis. Abundant evidence indicates that lncRNAs play an important role in these biological processes of adipose tissue accumulation. Many lncRNAs have been shown to influence adipocyte differentiation; for example, lncRNA_000414 inhibits the proliferation of intramuscular adipocytes [49]. Lnc-U90926 was found to be predominantly expressed in adipose tissue and was mainly located in the cytoplasm. The expression of Lnc-U90926 inhibited 3T3-L1 preadipocyte differentiation to adipose tissue by decreasing the mRNA levels of PPAR $\gamma 2$, adipose tissue acid binding protein 4 (FABP4), and adiponectin (AdipoQ), and the protein levels of PPAR $\gamma$ and FABP4 [50].

On the other hand, lipid metabolism can be considered an essential factor affecting adipose tissue deposition. Numerous IncRNAs have been recognized as a novel regulators of fat metabolism. A recent study showed that the lncRNA SRA limits adipose triglyceride lipase promoter activity in hepatic steatosis primarily by inhibiting forkhead box protein $\mathrm{O} 1$ expression [51]. The overexpression of $\ln \mathrm{HR} 1$ blocked the expression of sterol regulatory element binding protein (SREBP1c) and fatty acid synthase and suppressed the accumulation of triglycerides and lipid droplets in hepatocytes induced by oleic acid [52]. Evidence also showed that $\operatorname{lncSHGL}$ could recruit heterogeneous nuclear ribonucleoprotein A1 to increase the level of calmodulin protein without affecting its transcription. Thus, $\operatorname{lncSHGL}$ activated the phosphatidylinositol 3-kinase (PI3K)/Akt pathway and inhibited the mammalian target of rapamycin (mTOR)/SREBP-1C pathway to regulate hepatic glucose/lipid metabolism [53].

Although only a few functional lncRNAs have been well characterized so far, they have been shown to regulate the majority of gene expression programs in adipose tissue deposition [54]. Moreover, IncRNAs also regulate chromatinmodifying proteins, transcription, and translation processes. There are four archetypes of lncRNA functions, as detailed below. In addition, studies also have shown that lncRNAs may fulfill several archetypes to regulate adipose tissue accumulation (Table 1).

\section{Signal}

The first mode of action of lncRNAs is to regulate the transcription of downstream genes as molecular signals. LncRNAs can act as molecular signals because their expression is more cell-, tissue- and developmental stage-specific. The transcription of individual lncRNAs occurs at a very specific time and place to integrate developmental cues, interpret cellular context, or respond to diverse stimuli. Many studies have shown that $\operatorname{lncRNAs}$ can act as signals to regulate adipocytes differentiation and lipid metabolism. The lncRNA miR-31 host gene contributes to histone $\mathrm{H} 3$ lysine 4 trimethylation (H3K4me3) and $\mathrm{H} 3$ acetylation to promote the transcription of FABP4, which is an important molecule in the induction of adipocyte differentiation [55]. Similarly, it has been reported that the lncRNA adipogenic differentiation-induced noncoding RNA (ADINR) can increase H3K4me3 and decrease histone 3 lysine 27 trimethylation (H3K27me3) histone modifications in the $\mathrm{C} / \mathrm{EBPa}$ locus during adipogenesis by specifically binding to PA1 and recruiting MLL3/4 histone methyltransferase complexes. This indicated that ADINR might play vital roles in regulating the differentiation of human mesenchymal stem cells (MSCs) into adipocytes by modulating C/ $\mathrm{EBPa}$ in cis [56]. These findings suggest that lncRNAs are involved in the expression of adipose tissue-related genes through the regulation of histones (Figure 1A). In addition, lncRNAs can regulate the transcription factors associated with adipose tissue deposition by binding to their promoters of these transcription factors. For example, bovine adipocyte differentiation-related long noncoding RNA 1 significantly 
Table 1. LncRNAs function in adipose tissue deposition

\begin{tabular}{|c|c|c|c|c|}
\hline $\begin{array}{l}\text { Function } \\
\text { archetype }\end{array}$ & $\begin{array}{l}\text { LncRNA } \\
\text { acronym }\end{array}$ & Full name & Function & References \\
\hline \multirow[t]{3}{*}{ Signal } & MIR31HG & miRNA-31 host gene & Contribute to $\mathrm{H} 3 \mathrm{~K} 4 \mathrm{me} 3$ and $\mathrm{H} 3$ acetylation & {$[55]$} \\
\hline & ADINR & $\begin{array}{l}\text { Adipogenic differentiation } \\
\text { induced noncoding RNA }\end{array}$ & Function in histone modification during adipogenesis & {$[56]$} \\
\hline & BADLNCR1 & $\begin{array}{l}\text { Bovine adipocyte differentiation } \\
\text { related long non-coding RNA } 1\end{array}$ & Inhibit mitochondrial protein GLRX5 promoter activity & {$[57]$} \\
\hline & GAS5 & Growth arrest specific 5 & Regulate adipocyte differentiation & [14] \\
\hline & ADNCR & $\begin{array}{l}\text { Adipocyte differentiation- associated } \\
\text { long noncoding RNA }\end{array}$ & Inhibit adipocyte differentiation by regulating SIRT1 & {$[58]$} \\
\hline & TINCR & $\begin{array}{l}\text { Terminal differentiation-induced } \\
\text { ncRNA }\end{array}$ & $\begin{array}{l}\text { Modulate adipocyte differentiation of adipose } \\
\text { tissue-derived MSCs }\end{array}$ & [59] \\
\hline Guide & ADINR & $\begin{array}{l}\text { Adipogenic differentiation induced } \\
\text { noncoding RNA }\end{array}$ & $\begin{array}{l}\text { Activate } \mathrm{C} / \mathrm{EBPa} \text { transcription in cis to promote } \\
\text { adipogenesis }\end{array}$ & {$[56]$} \\
\hline
\end{tabular}

inhibited the enhanced expression of mitochondrial protein glutaredoxin 5 promoter activity through $\mathrm{C} / \mathrm{EBPa}$ [57] (Figure 1B). These studies demonstrated that $\operatorname{lncRNAs}$ can act as molecular signals by directly regulating histone modification or the promoters of transcription factors. In addition, these lncRNAs can serve as biomarkers of functionally significant biological events because the chromatin state and the expression of regulatory elements can be easily inferred by the expression of their related lncRNAs. Chromatin immunoprecipitation (ChIP) and chromatin isolation by RNA purification (CHIRP) assays are generally required for the functional verification of these lncRNAs in the modulation of histones.

\section{Decoy}

The second action mode of lncRNAs is interference. LncRNAs can directly bind to transcription factors to then regulate downstream gene transcription. This mainly represents a molecular decoy mechanism, in which the lncRNA acts as a "molecular sink" for the transcription factors. LncRNAs acting as decoys of miRNAs have been called competing endogenous RNAs (ceRNAs), and the ceRNA mechanism is a theoretical hypothesis of RNA expression regulation. Endogenous IncRNAs can act as ceRNAs by competing with the seed sequences of miRNAs to release related mRNAs from miRNA-mediated inhibition. This is also the most common pattern of IncRNA interaction with each other [63] (Figure 2). For example, LRP6 is the direct target of $m i R-21$ and is required for $m i R-21$-induced intracellular lipid accumulation. Research has shown that the lncRNA maternally expressed gene 3 (lncRNA MEG3) is considered a ceRNA that regulates hepatic lipogenesis by competitively

\section{LncRNA}
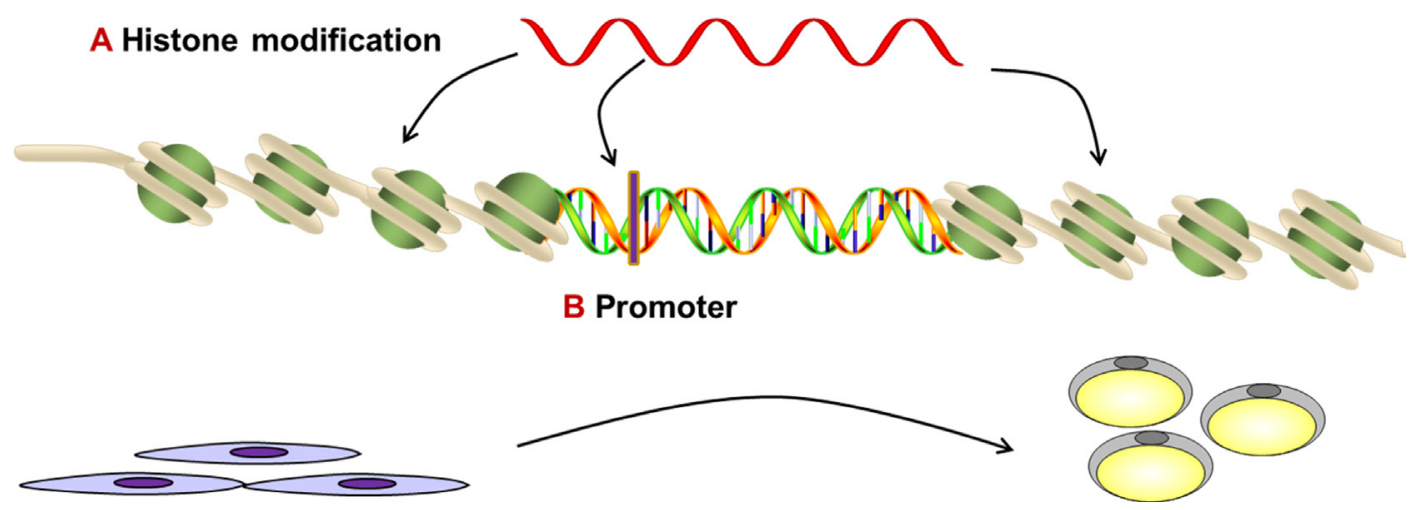

Figure 1. Long noncoding RNAs regulate the expression of adipogenic genes by modifying of chromatin. 


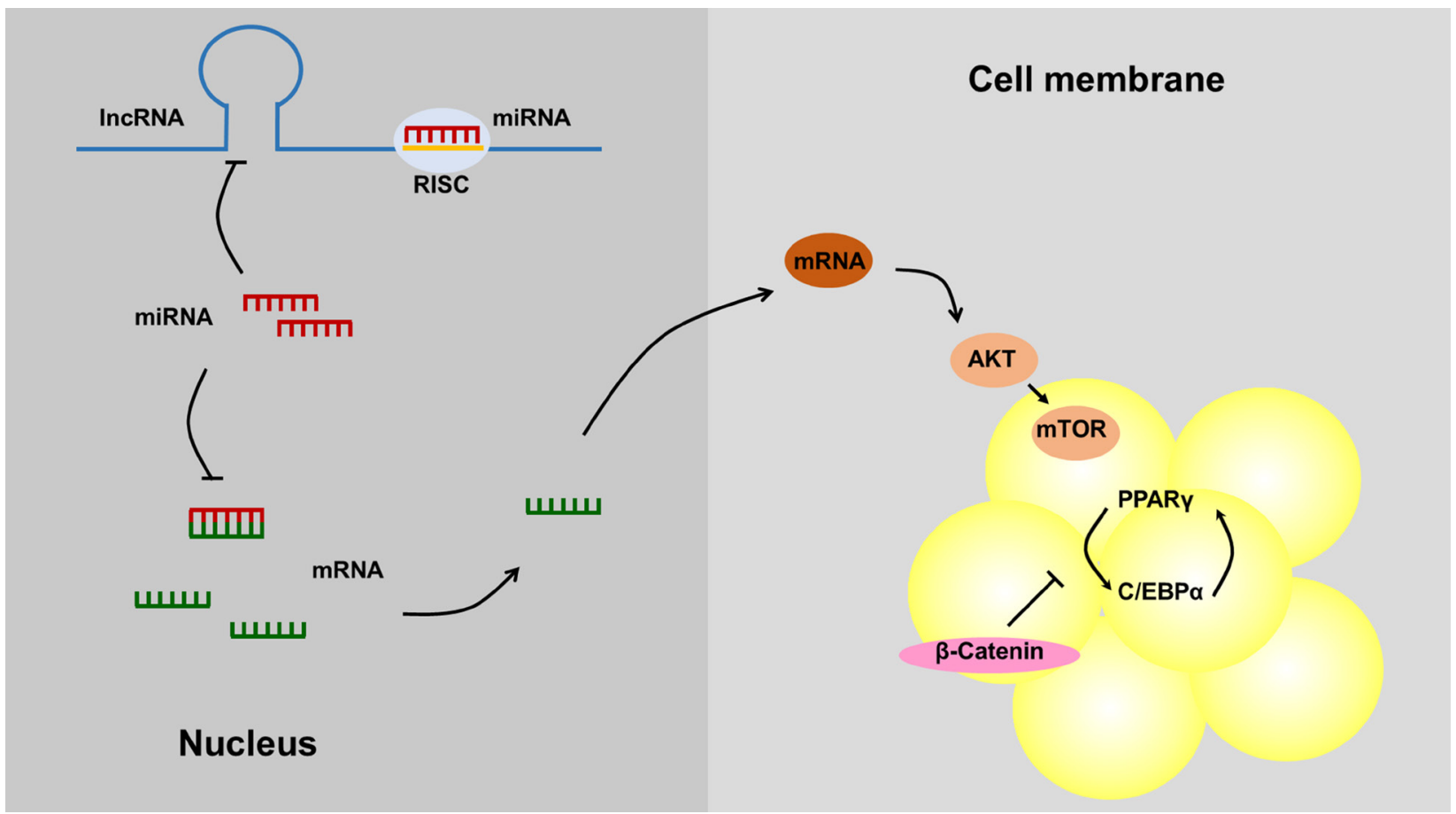

Figure 2. LncRNAs act as ceRNA to regulate adipogenesis. LncRNAs competitively bind to miRNAs, which increases levels of the mRNAs originally bound to miRNAs and thus regulates adipose tissue deposition (IncRNA expression in the nucleus is indicated as an example). LncRNAs, long noncoding RNAs; ceRNA, competing endogenous RNAs; miRNAs, microRNAs; mRNAs, messenger RNA.

binding to miR-21 with LRP6 through the mTOR signaling pathway to alleviate lipid over-deposition [15]. The LncRNA growth arrest-specific transcript 5 can regulate the adipogenic differentiation of MSCs by acting as a ceRNA to sponge miR-18a [14]. Similarly, adipocyte differentiation-associated long noncoding RNA could act as a ceRNA to interact with miR-204 and inhibit adipocyte differentiation by regulating sirtuin 1 [58]. The LncRNA terminal differentiation-induced ncRNA also served as a sponge of miR-31, which can directly target $\mathrm{C} / \mathrm{EBP} \alpha$ to modulate adipogenic differentiation in human adipose tissue-derived from MSCs [59].

\section{Guide}

Third, lncRNAs act as guides to bind proteins and then direct the localization of the ribonucleoprotein complex to specific targets. This transcriptional regulation mediated by $\operatorname{lncRNAs}$ can be cis-acting or trans-acting (Figure 3). Evidence has shown that ADINR can specifically bind to PA1, recruiting MLL3/4 histone methyltransferase complexes and then activating $\mathrm{C} /$ EBPa transcription in cis to promote adipogenesis [56]. Heterogeneous nuclear ribonucleoprotein $\mathrm{U}$ (hnRNPU) is required for brown adipocyte development, and brown adipose tissuespecific lncRNA 1 (lnc BATE1) can interact with hnRNPU to modulate brown adipogenesis by acting in trans [60].

\section{Scaffold}

Finally, lncRNAs have different binding domains for different effector molecules, which makes it possible to bind multiple effectors simultaneously. Thus, IncRNAs could induce transcriptional activation or inhibition in time and space by interacting with different effectors. Scaffolds might be the most functionally intricate and complex regulatory mechanism of $\operatorname{lncRNAs,~in~which~lncRNAs~act~as~central~platforms~}$ to affect the regulation of multiple signaling pathways (Figure 4). $H 19$ (a lncRNA transcribed from the H19 gene) is a classic lncRNA that regulates ligand-dependent corepressor to

\section{ribonucleoprotein complex}

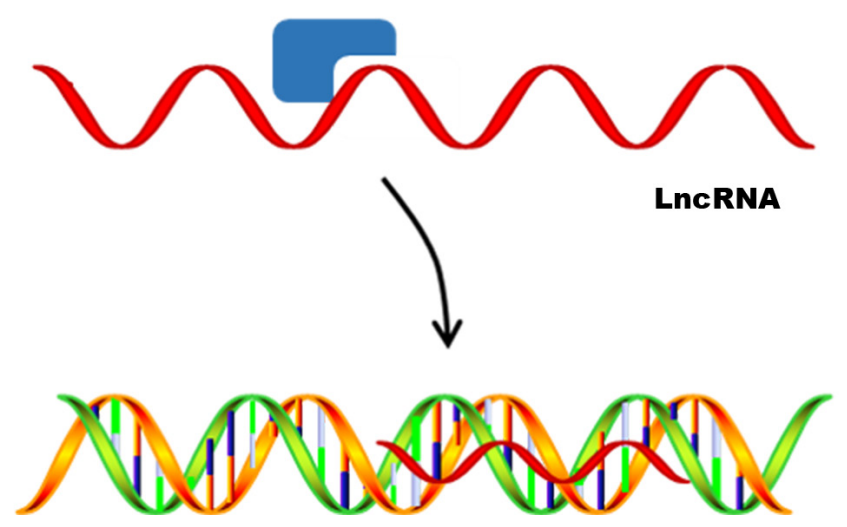

Figure 3. Long noncoding RNAs act as a guide to direct the localization of ribonucleoprotein complexes to specific targets. 
regulate the balance between osteogenic and adipogenic differentiation of bone marrow stromal stem cells in mice by sponging miR-188 [61]. In addition, H19 can bind with methyltransferase MBD1 to form H19-MBD1 complexes in mature brown adipocytes, potentially via MBD1-dependent alterations of $\mathrm{H} 3 \mathrm{~K} 9 \mathrm{me} 3 \mathrm{KMT}$ recruitment. In these two studies, $H 19$ was proposed as a paternally expressed gene (PEG) gatekeeper in brown adipocytes and was shown to repress brown adipose tissues PEG by recruiting MBD1 chromatin modifiers [62].

\section{STUDIES OF LncRNAs IN ADIPOSE TISSUE DEPOSITION OF LIVESTOCK}

Compared with clinical studies in humans, studies of the function of $\operatorname{lncRNAs}$ in adipose tissue deposition and meat quality in livestock have been limited. Many transcriptome sequencing studies on adipose tissue accumulation in different species of livestock have been published. However, most of these studies focused on screening differentially expressed genes (DEGs) using RNA-seq without sufficient exploration of the function and mechanisms of the DEGs. In previous papers, a number of lncRNAs have been well characterized to be involved in adipose tissue accumulation in cells or rodents $[50,64]$. These studies could provide insights into the important role and application of lncRNAs in regulating the development of adipose tissue in farm animals. Although the conservation of lncRNAs is lower than that of other ncRNAs and protein-coding RNAs, the functions of lncRNAs are important [65]. Thus, studying the conservation of lncRNAs among different species seems to be significant for farm animal genomic and transcriptomic studies, such as those focusing on adipose tissue deposition and fat metabolism. When a certain lncRNA is well conserved in different species such as humans, mice, pigs, and cattle, the known mechanism of action and function of this lncRNA might be applicable to other animals. Therefore,

\section{ribonucleoprotein complex}

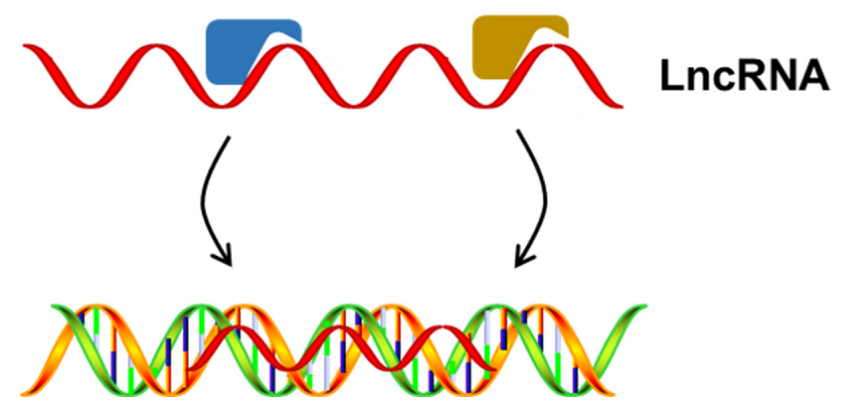

Figure 4. Long noncoding RNAs act as scaffolds that bring together multiple proteins to form ribonucleoprotein complexes. research on lncRNA conservation among species might be valuable for the research or application of conserved lncRNAs in different animals, especially domestic large livestock animals.

Cattle

Intramuscular fat deposition in cattle is of economic importance and has been explored in several researches. In livestock, adipose tissue and muscle development are closely related to body weight, marbling content, and meat quality, especially marble beef yield [66]. High-throughput sequencing was performed on six calf and adult bovine adipose tissues from Chinese Qinchuan cattle to select candidate lncRNAs associated with adipose tissue accumulation and muscle development [67]. The researchers also focused on identifying DEGs between muscle and adipose tissues to determine their effects on intramuscular fat and meat quality. Hanwoo cattle (Bos taurus coreanae) are famous for thier high marbling content and relatively thin muscle fibers in Korea. A previous study profiled tissue-specific lncRNAs by using comparative analysis of muscle and other adipose tissues (intramuscular, subcutaneous, and omental) in Hanwoo cattle, and $76 \operatorname{lncRNAs}$ were identified to be associated with marbling content and meat quality [68]. In livestock production, a novel lncRNA mainly localized to the nucleus and named miR-221 host gene (MIR221HG) because it overlaps with miR-221 in the genome was proven to regulate bovine adipocyte differentiation [69]. Studies found that the expression of lnc FAM200B in bovines had a significant positive correlation with $C / E B P \alpha$ expression, suggesting that lnc FAM $200 B$ might participate in the regulation of adipocyte development [70]. In addition, evidence indicated that $P S X V-9$, a novel antisense lncRNA of $C / E B P \alpha$, could inhibit bovine adipogenic differentiation [71].

\section{Pigs}

Domestic pigs (Sus scrofa domesticus) are one of the major animal models of obesity and adipose tissue deposition [63,72]. Similar to studies on lncRNAs in cattle, there are also numerous high-throughput sequencing studies comparing different pig species. For example, researchers found DEGs between Jinhua and Landrace pigs [73] and Laiwu and Large White pigs [74] and screened candidate lncRNAs associated with adipose tissue accumulation. Evidence has shown that the lncRNA IMF4 regulates porcine intramuscular preadipocyte adipogenesis by attenuating autophagy to inhibit lipolysis [18]. PU.1, also known as spleen focus forming virus proviral integration oncogene spil, inhibits adipogenesis. In addition, the PU.1 antisense lncRNA (PU.1 AS lncRNA) promotes adipogenesis through the formation of a sense-anti-sense RNA duplex with PU.1 mRNA [75]. Similarly, PLA2G16-AS and PLA2G16 were also reported to be expressed in pork adipose tissues [76]. Besides, the lncRNA IMFlnc1, a differentially 
expressed lncRNA identified through sequencing between Huainan and large white pigs, promoted porcine intramuscular adipocyte adipogenesis by sponging miR-199a-5p and upregulating caveolin-1 expression [77].

\section{Other livestock}

There have also been abundant studies about adipose tissue deposition based on high-throughput sequencing data from chickens [78], rabbits [79], sheep [80] and other kinds of livestock. Evidence also showed that the lncRNA intramuscular fat-associated long non-coding RNA promoted intramuscular adipocyte differentiation by sponging miR-128-3p and miR-27b-3p in chicken which targeted PPAR $\gamma$ [81]. Although these studies have identified differentially expressed candidate lncRNAs related to adipose tissue accumulation, there are still some drawbacks. First, these studies used experimental samples that might not have been considered the genetic background. In addition, the screened differentially expressed lncRNAs were not well explored, and the mechanism of regulation of adipose tissue deposition by these potential $\operatorname{lncRNAs}$ is unclear. Therefore, research methods to explore lncRNA functions in adipose tissue accumulation have important future implications.

\section{PERSPECTIVES AND FUTURE OPPORTUNITIES}

To date, differential adipose tissue deposition abilities have been observed in different breeds and even the different individuals under the same genetic background, and the mechanism of this phenomenon is still unknown. The key developmental period and physiological and biochemical mechanisms of adipose tissue accumulation still need to be further explored. In addition, research on the interaction and regulation between muscle and intramuscular adipose tissue is an important aspect to improve the meat quality of livestock production. It is essential to explore this regulatory mechanism for its implementation and application in meat production in order to improve livestock productivity and the development of high-quality ecological products.

Compared to methods involving biologics or small molecules, RNA-based breeding and production are characterized by unique and important benefits and challenges that should be taken into consideration. RNA methods are based on nucleotide hybridization to interact with the targets and include identification of the sequence and testing of candidate genes for activity. Despite these advantages, mechanisms of lncRNA regulation of adipose tissue deposition have to be characterized and optimized, which can often be a challenging process. Although lncRNA applications in farm animals are still at an early stage, it is expected that production based on the involvement of lncRNAs in adipose tissue deposition and pathway regulation will one day be achieved. Expanded lncRNA research in livestock species will provide new fundamental insights into the complexity of gene regulation of important biological processes. For the identification of candidate biomarkers, focusing on lineage-specific lncRNAs seems to be the ideal approach, as is the case with the lncRNA MEG3 mentioned above [15]. Therefore, researchers should pay attention to the conservation of $\ln$ CRNAs before starting experiments. In addition, the level of lncRNA expression is also a key factor for developing lncRNA based predictions. Overall, lncRNAs are a promising tool that can enable researchers to discover molecular signatures that help to improve meat quality for fam animals in the future. Given the conservation and specific expression of $\operatorname{lncRNAs}$ and its response to adipose tissue deposition, the lncRNA class deserves further investigation into their physiological roles and molecular mechanisms of action, which finally, will contribute to better explain the whole phenotypic variation of adipose tissue accumulation and basic biological processes in livestock animals.

\section{AUTHOR CONTRIBUTION}

Conceptualization, L.W., Y.X., Y.Z.; writing-original draft preparation, L.W.; writing-review and editing, L.W., Y.X., Y.Z.; visualization, L.W., W.C.; supervision, Y.Z. All authors have read and agreed to the published version of the manuscript.

\section{CONFLICT OF INTEREST}

We certify that there is no conflict of interest with any financial organization regarding the material discussed in the manuscript.

\section{FUNDING}

This study was supported financially by the Agricultural Animal Breeding Project of Shandong Province (No. 2020 LZGC012), Funds of Shandong "Double Tops" Program (No. SYL2017YSTD12), Shandong Modern Pig Technology \& Industry System Project (No. SDAIT-08-02), Shandong Provincial Natural Science Foundation (No. ZR2018BC046, ZR2019MC053).

\section{REFERENCES}

1. Dodson MV, Jiang Z, Chen J, et al. Allied industry approaches to alter intramuscular fat content and composition in beef animals. J Food Sci 2010;75:R1-R8. https://doi.org/10.1111/j. 1750-3841.2009.01396.x

2. Corbin $\mathrm{CH}$, O'Quinn TG, Garmyn AJ, et al. Sensory evalu- 
ation of tender beef strip loin steaks of varying marbling levels and quality treatments. Meat Sci 2015;100:24-31. https:// doi.org/10.1016/j.meatsci.2014.09.009

3. Caprio S, Perry R, Kursawe R. Adolescent obesity and insulin resistance: roles of ectopic fat accumulation and adipose inflammation. Gastroenterology 2017;152:1638-46. https:// doi.org/10.1053/j.gastro.2016.12.051

4. Neeland IJ, Ross R, Després J, et al. Visceral and ectopic fat, atherosclerosis, and cardiometabolic disease: a position statement. Lancet Diabetes Endocrinol 2019;7:715-25. https:// doi.org/10.1016/S2213-8587(19)30084-1

5. Tang QQ, Lane MD. Adipogenesis: from stem cell to adipocyte. Annu Rev Biochem 2012;81:715-36. https://doi.org/10.1146/ annurev-biochem-052110-115718

6. Rosen ED, Walkey CJ, Puigserver P, Spiegelman BM. Transcriptional regulation of adipogenesis. Gene Dev 2000;14: 1293-307.

7. Guiu Jurado E, Unthan M, Böhler N, et al. Bone morphogenetic protein 2 (BMP2) may contribute to partition of energy storage into visceral and subcutaneous fat depots. Obesity 2016;24:2092-2100. https://doi.org/10.1002/oby. 21571

8. Hardouin P, Rharass T, Lucas S. Bone marrow adipose tissue: to be or not to be a typical adipose tissue? Front Endocrinol 2016;7:85. https://doi.org/10.3389/fendo.2016.00085

9. Hausman GJ, Basu U, Du M, Fernyhough-Culver M, Dodson MV. Intermuscular and intramuscular adipose tissues: bad vs. Good adipose tissues. Adipocyte 2014;3:242-55. https:// doi.org/10.4161/adip.28546

10. Newcom DW, Baas TJ, Schwab CR, Stalder KJ. Genetic and phenotypic relationships between individual subcutaneous backfat layers and percentage of longissimus intramuscular fat in duroc swine. J Anim Sci 2005;83:316-23. https://doi. org/10.2527/2005.832316x

11. Hamdy O, Porramatikul S, Al-Ozairi E. Metabolic obesity: the paradox between visceral and subcutaneous fat. Curr Diabetes Rev 2006;2:367-73. https://doi.org/10.2174/15733 99810602040367

12. Xu L, Ma X, Verma NK, et al. Ablation of PPAR $\gamma$ in subcutaneous fat exacerbates age-associated obesity and metabolic decline. Aging Cell 2018;17:e12721. https://doi.org/10.1111/ acel.12721

13. Bouchi R, Takeuchi T, Akihisa M, et al. High visceral fat with low subcutaneous fat accumulation as a determinant of atherosclerosis in patients with type 2 diabetes. Cardiovasc Diabetol 2015;14:136. https://doi.org/10.1186/s12933-0150302-4

14. Li M, Xie Z, Wang P, et al. The long noncoding RNA GAS5 negatively regulates the adipogenic differentiation of MSCs by modulating the miR-18a/CTGF axis as a ceRNA. Cell Death Dis 2018;9:554. https://doi.org/10.1038/s41419-0180627-5
15. Huang P, Huang F, Liu H, Zhang T, Yang M, Sun C. LncRNA MEG3 functions as a ceRNA in regulating hepatic lipogenesis by competitively binding to miR-21 with LRP6. Metabolism 2019;94:1-8. https://doi.org/10.1016/j.metabol.2019.01.018

16. Cai B, Li Z, Ma M, et al. LncRNA-Sixl encodes a micropeptide to activate Six 1 in cis and is involved in cell proliferation and muscle growth. Front Physiol 2017;8:230. https://doi. org/10.3389/fphys.2017.00230

17. Huang J, Zheng Q, Wang S, Wei X, Li F, Ma Y. High-throughput RNA sequencing reveals NDUFC2-AS IncRNA promotes adipogenic differentiation in chinese buffalo (Bubalus bubalis L.). Genes-Basel 2019;10:689. https://doi.org/10.3390/genes 10090689

18. Sun Y, Cai R, Wang Y, Zhao R, Qin J, Pang W. A newly identified lncrna lncIMF4 controls adipogenesis of porcine intramuscular preadipocyte through attenuating autophagy to inhibit lipolysis. Animals 2020;10:926. https://doi.org/10.3390/ ani10060926

19. Weikard R, Demasius W, Kuehn C. Mining long noncoding RNA in livestock. Anim Genet 2017;48:3-18. https://doi.org/ 10.1111/age.12493

20. Kosinska-Selbi B, Mielczarek M, Szyda J. Long non-coding RNA in livestock. Animal 2020;14:2003-13. https://doi.org/ 10.1017/S1751731120000841

21. Diederichs S. The four dimensions of noncoding RNA conservation. Trends Genet 2014;30:121-3. https://doi.org/10. 1016/j.tig.2014.01.004

22. Laurent GS, Wahlestedt C, Kapranov P. The landscape of long noncoding RNA classification. Trends Genet 2015;31: 239-51. https://doi.org/10.1016/j.tig.2015.03.007

23. Guttman M, Amit I, Garber M, et al. Chromatin signature reveals over a thousand highly conserved large non-coding RNAs in mammals. Nature 2009;458:223-7. https://doi.org/ 10.1038/nature07672

24. Chen YG, Satpathy AT, Chang HY. Gene regulation in the immune system by long noncoding RNAs. Nat Immunol 2017;18:962-72. https://doi.org/10.1038/ni.3771

25. Ulitsky I, Shkumatava A, Jan CH, Sive H, Bartel DP. Conserved function of lincRNAs in vertebrate embryonic development despite rapid sequence evolution. Cell 2011;147:1537-50. https://doi.org/10.1016/j.cell.2011.11.055

26. Carninci P, Kasukawa T, Katayama S, et al. The transcriptional landscape of the mammalian genome. Science 2005; 309:1559-63. https://doi.org/10.1126/science.1112014

27. Somarowthu S, Legiewicz M, Chillón I, Marcia M, Liu F, Pyle AM. HOTAIR forms an intricate and modular secondary structure. Mol Cell 2015;58:353-61. https://doi.org/10.1016/ j.molcel.2015.03.006

28. Liu F, Somarowthu S, Pyle AM. Visualizing the secondary and tertiary architectural domains of IncRNA Repa. Nat Chem Biol 2017;13:282-9. https://doi.org/10.1038/nchembio. 2272 
29. Quinn JJ, Zhang QC, Georgiev P, Ilik IA, Akhtar A, Chang HY. Rapid evolutionary turnover underlies conserved lncRNAgenome interactions. Gene Dev 2016;30:191-207. https://doi. org/10.1101/gad.272187.115

30. Graf J, Kretz M. From structure to function: route to understanding lncRNA mechanism. Bioessays 2020;42:2000027. https://doi.org/10.1002/bies.202000027

31. Derrien T, Johnson R, Bussotti G, et al. The GENCODE v7 catalog of human long noncoding RNAs: analysis of their gene structure, evolution, and expression. Genome Res 2012; 22:1775-89. https://doi.org/10.1101/gr.132159.111

32. Cabili MN, Trapnell C, Goff L, et al. Integrative annotation of human large intergenic noncoding RNAs reveals global properties and specific subclasses. Gene Dev 2011;25:191527. https://doi.org/10.1101/gad.17446611

33. Flynn RA, Chang HY. Long noncoding RNAs in cell-fate programming and reprogramming. Cell Stem Cell 2014;14: 752-61. https://doi.org/10.1016/j.stem.2014.05.014

34. Latorre J, Fernández-Real JM. LncRNAs in adipose tissue from obese and insulin-resistant subjects: new targets for therapy? Ebiomedicine 2018;30:10-1. https://doi.org/10.1016/ j.ebiom.2018.03.023

35. Gupta PK. Competing endogenous RNA (ceRNA): a new class of RNA working as miRNA sponges. Curr Sci India 2014;106:823-9.

36. Cabili MN, Dunagin MC, Mcclanahan PD, et al. Localization and abundance analysis of human lncRNAs at single-cell and single-molecule resolution. Genome Biol 2015;16:20. https://doi.org/10.1186/s13059-015-0586-4

37. Cristancho AG, Lazar MA. Forming functional fat: a growing understanding of adipocyte differentiation. Nat Rev Mol Cell Biol 2011;12:722-34. https://doi.org/10.1038/nrm3198

38. Cawthorn WP, Scheller EL, Macdougald OA. Adipose tissue stem cells meet preadipocyte commitment: going back to the future. J Lipid Res 2012;53:227-46. https://doi.org/10. 1194/jlr.R021089

39. Huang $\mathrm{H}$, Song T, Li X, et al. BMP signaling pathway is required for commitment of $\mathrm{C} 3 \mathrm{H} 10 \mathrm{~T} 1 / 2$ pluripotent stem cells to the adipocyte lineage. Proc Natl Acad Sci USA 2009;106: 12670-5. https://doi.org/10.1073/pnas.0906266106

40. Louveau I, Perruchot M, Bonnet M, Gondret F. Invited review: Pre-and postnatal adipose tissue development in farm animals: from stem cells to adipocyte physiology. Animal 2016;10: 1839-47. https://doi.org/10.1017/S1751731116000872

41. Fang X, Stroud MJ, Ouyang K, et al. Adipocyte-specific loss of PPAR $\gamma$ attenuates cardiac hypertrophy. JCI Insight 2016; 1:e89908. https://doi.org/10.1172/jci.insight.89908

42. Smith A, Yu X, Yin L. Diazinon exposure activated transcriptional factors CCAAT-enhancer-binding proteins $\alpha(\mathrm{C} / \mathrm{EBP} \alpha)$ and peroxisome proliferator-activated receptor $\gamma(\operatorname{PPAR} \gamma)$ and induced adipogenesis in 3T3-L1 preadipocytes. Pestic Biochem Physiol 2018;150:48-58. https://doi.org/10.1016/ j.pestbp.2018.07.003

43. Oishi Y, Manabe I, Tobe K, et al. Krüppel-like transcription factor KLF5 is a key regulator of adipocyte differentiation. Cell Metab 2005;1:27-39. https://doi.org/10.1016/j.cmet.2004. 11.005

44. Zhang JW, Klemm DJ, Vinson C, Lane MD. Role of creb in transcriptional regulation of CCAAT/enhancer-binding protein $\beta$ gene during adipogenesis. J Biol Chem 2004;279: 4471-8. https://doi.org/10.1074/jbc.M311327200

45. Li S, Wu J. TGF- $\beta /$ SMAD signaling regulation of mesenchymal stem cells in adipocyte commitment. Stem Cell Res Ther 2020;11:41. https://doi.org/10.1186/s13287-020-1552-y

46. Kanazawa A, Tsukada S, Kamiyama M, Yanagimoto T, Nakajima M, Maeda S. Wnt5b partially inhibits canonical Wnt/ $\beta$-catenin signaling pathway and promotes adipogenesis in 3T3-L1 preadipocytes. Biochem Biophys Res Commun 2005; 330:505-10. https://doi.org/10.1016/j.bbrc.2005.03.007

47. Chen J, Bao C, Kim JT, Cho JS, Qiu S, Lee HJ. Sulforaphene inhibition of adipogenesis via Hedgehog signaling in 3T3-L1 adipocytes. J Agric Food Chem 2018;66:11926-34. https:// doi.org/10.1021/acs.jafc.8b04330

48. Song B, Chi Y, Li X, et al. Inhibition of Notch signaling promotes the adipogenic differentiation of mesenchymal stem cells through autophagy activation and PTEN-PI3K/AKT/ mTOR pathway. Cell Physiol Biochem 2015;36:1991-2002. https://doi.org/10.1159/000430167

49. Sun Y, Chen X, Qin J, Liu S, Zhao R. Comparative analysis of long noncoding RNAs expressed during intramuscular adipocytes adipogenesis in fat-type and lean-type pigs. J Agric Food Chem 2018;66:12122-30. https://doi.org/10.1021/ acs.jafc. 8 b0 04243

50. Chen J, Liu Y, Lu S, et al. The role and possible mechanism of lncRNA U90926 in modulating 3T3-L1 preadipocyte differentiation. Int J Obes 2017;41:299-308. https://doi.org/ 10.1038/ijo.2016.189

51. Chen G, Yu D, Nian X, et al. LncRNA SRA promotes hepatic steatosis through repressing the expression of adipose triglyceride lipase (ATGL). Sci Rep-Uk 2016;6:35531. https:// doi.org/10.1038/srep35531

52. Duan L, Min C, Niu Y, et al. Identification of a novel human long non-coding RNA that regulates hepatic lipid metabolism by inhibiting SREBP-1c. Int J Biol Sci 2017;13:349-57. https:// doi.org/10.7150/ijbs.16635

53. Wang J, Yang W, Chen Z, et al. Long noncoding RNA incSHGL recruits hnRNPA1 to suppress hepatic gluconeogenesis and lipogenesis. Diabetes 2018;67:581-93. https://doi.org/10.2337/ db17-0799

54. Wapinski O, Chang HY. Long noncoding RNAs and human disease. Trends Cell Biol 2011;21:354-61. https://doi.org/10. 1016/j.tcb.2011.04.001

55. Huang Y, Jin C, Zheng Y, et al. Knockdown of lncRNA MIR31HG inhibits adipocyte differentiation of human adipose- 
derived stem cells via histone modification of FABP4. Sci Rep-Uk 2017;7:8080. https://doi.org/10.1038/s41598-01708131-6

56. Xiao T, Liu L, Li H, et al. Long noncoding RNA ADINR regulates adipogenesis by transcriptionally activating $C / E B P \alpha$. Stem Cell Rep 2015;5:856-65. https://doi.org/10.1016/j.stemcr. 2015.09.007

57. Cai H, Li M, Jian W, et al. A novel lncRNA BADLNCR1 inhibits bovine adipogenesis by repressing GLRX 5 expression. J Cell Mol Med 2020;24:7175-86. https://doi.org/10.1111/jcmm. 15181

58. Li M, Sun X, Cai H, et al. Long non-coding RNA ADNCR suppresses adipogenic differentiation by targeting miR-204. Biochim Biophys Acta Gene Regul Mech 2016;1859:871-82. https://doi.org/10.1016/j.bbagrm.2016.05.003

59. Liu Y, Wang Y, He X, et al. LncRNA TINCR/miR-31-5p/C/ EBP- $\alpha$ feedback loop modulates the adipogenic differentiation process in human adipose tissue-derived mesenchymal stem cells. Stem Cell Res 2018;32:35-42. https://doi.org/10.1016/ j.scr.2018.08.016

60. Alvarez-Dominguez JR, Bai Z, Xu D, et al. De novo reconstruction of adipose tissue transcriptomes reveals long noncoding RNA regulators of brown adipocyte development. Cell Metab 2015;21:764-76. https://doi.org/10.1016/j.cmet. 2015.04.003

61. Wang Y, Liu W, Liu Y, et al. Long noncoding RNA H19 mediates $L C o R$ to impact the osteogenic and adipogenic differentiation of mBMSCs in mice through sponging miR-188. J Cell Physiol 2018;233:7435-46. https://doi.org/10.1002/jcp. 26589

62. Schmidt E, Dhaouadi I, Gaziano I, et al. LincRNA H19 protects from dietary obesity by constraining expression of monoallelic genes in brown fat. Nat Commun 2018;9:3622. https://doi.org/10.1038/s41467-018-05933-8

63. Salmena L, Poliseno L, Tay Y, Kats L, Pandolfi PP. A ceRNA hypothesis: the Rosetta Stone of a hidden RNA language? Cell 2011;146:353-8. https://doi.org/10.1016/j.cell.2011.07. 014

64. Ma M, Duan R, Shen L, et al. The lncRNA Gm15622 stimulates SREBP-1c expression and hepatic lipid accumulation by sponging the miR-742-3p in mice. J Lipid Res 2020;61:105264. https://doi.org/10.1194/jlr.RA120000664

65. Johnsson P, Ackley A, Vidarsdottir L, et al. A pseudogene long-noncoding-RNA network regulates PTEN transcription and translation in human cells. Nat Struct Mol Biol 2013;20: 440-6. https://doi.org/10.1038/nsmb.2516

66. Pethick DW, Harper GS, Oddy VH. Growth, development and nutritional manipulation of marbling in cattle: a review. Aust J Exp Agric 2004;44:705-15.

67. Jiang R, Li H, Huang Y, Lan X, Lei C, Chen H. Transcriptome profiling of lncRNA related to fat tissues of qinchuan cattle. Gene 2020;742:144587. https://doi.org/10.1016/j.gene.2020.
144587

68. Choi JY, Shin D, Lee HJ, Oh JD. Comparison of long noncoding RNA between muscles and adipose tissues in Hanwoo beef cattle. Anim Cells Syst 2019;23:50-8. https://doi.org/10. 1080/19768354.2018.1512522

69. Li M, Gao Q, Tian Z, et al. MIR221HG is a novel long noncoding RNA that inhibits bovine adipocyte differentiation. Genes-Basel 2020;11:29. https://doi.org/10.3390/genes110 10029

70. Zhang S, Kang Z, Cai H, et al. Identification of novel alternative splicing of bovine lncrna $\operatorname{lncFAM200B}$ and its effects on preadipocyte proliferation. J Cell Physiol 2020;236:60111. https://doi.org/10.1002/jcp.29887

71. Li A, Hu Y, Liu X, Zhao L, Tian Q, Du M. PSXV-9 a novel anti-sense lncRNA of CEBPA inhibits bovine adipogenic differentiation. J Anim Sci 2018;96(Suppl 3):245. https:// doi.org/10.1093/jas/sky404.535

72. Chen M, Wang J, Wang Y, Wu Y, Fu J, Liu J. Genome-wide detection of selection signatures in chinese indigenous laiwu pigs revealed candidate genes regulating fat deposition in muscle. BMC Genet 2018;19:31. https://doi.org/10.1186/ s12863-018-0622-y

73. Miao Z, Wang S, Zhang J, et al. Identification and comparison of long non-conding RNA in Jinhua and Landrace pigs. Biochem Biophys Res Commun 2018;506:765-71. https:// doi.org/10.1016/j.bbrc.2018.06.028

74. Cui JX, Zeng QF, Chen W, Zhang H, Zeng YQ. Analysis and preliminary validation of the molecular mechanism of fat deposition in fatty and lean pigs by high-throughput sequencing. Mamm Genome 2019;30:71-80. https://doi.org/ 10.1007/s00335-019-09795-3

75. Wei N, Wang Y, Xu RX, et al. PU.1 antisense lnc RNA against its mRNA translation promotes adipogenesis in porcine preadipocytes. Anim Genet 2015;46:133-40. https://doi.org/ 10.1111/age. 12275

76. Liu P, Jin L, Zhao L, et al. Identification of a novel antisense long non-coding RNA PLA2G16-AS that regulates the expression of PLA2G16 in pigs. Gene 2018;671:78-84. https:// doi.org/10.1016/j.gene.2018.05.114

77. Wang J, Chen M, Chen J, et al. LncRNA IMFlnc1 promotes porcine intramuscular adipocyte adipogenesis by sponging miR-199a-5p to up-regulate CAV-1. BMC Mol Cell Biol 2020;21:77. https://doi.org/10.1186/s12860-020-00324-8

78. Muret K, Klopp C, Wucher V, et al. Long noncoding RNA repertoire in chicken liver and adipose tissue. Genet Sel Evol 2017;49:6. https://doi.org/10.1186/s12711-016-0275-0

79. Wang G, Du K, Hu S, et al. Genome-wide identification and characterization of long non-coding RNAs during postnatal development of rabbit adipose tissue. Lipids Health Dis 2018; 17:271. https://doi.org/10.1186/s12944-018-0915-1

80. Ma L, Zhang M, Jin Y, et al. Comparative transcriptome profiling of mRNA and lncRNA related to tail adipose tissues 
of sheep. Front Genet 2018;9:365. https://doi.org/10.3389/ fgene.2018.00365

81. Zhang M, Li F, Sun JW, et al. LncRNA IMFNCR promotes intramuscular adipocyte differentiation by sponging miR128-3p and miR-27b-3p. Front Genet 2019;10:42. https:// doi.org/10.3389/fgene.2019.00042 